OPEN ACCESS

Edited by:

Jihui Zhang,

The Chinese University of Hong

Kong, China

Reviewed by:

Chengyu $\mathrm{Li}$,

Beijing University of Chinese

Medicine, China

Kittisak Sawanyawisuth

Khon Kaen University, Thailand

*Correspondence:

Jingbo Zhai

zhaijingbo@foxmail.com

Hongtao Yang

tjtcmht@126.com

tThese authors have contributed equally to this work and share first

authorship

Specialty section: This article was submitted to

Sleep Disorders,

a section of the journal

Frontiers in Psychiatry

Received: 25 June 2020 Accepted: 17 June 2021

Published: 19 July 2021

Citation:

Pei M, Chen J, Dong S, Yang B, Yang $K$, Wei L, Zhai J and Yang $H$

(2021) Auricular Acupressure for Insomnia in Patients With

Maintenance Hemodialysis: A

Systematic Review and Meta-Analysis.

Front. Psychiatry 12:576050

doi: 10.3389/fpsyt.2021.576050

\section{Auricular Acupressure for Insomnia in Patients With Maintenance Hemodialysis: A Systematic Review and Meta-Analysis}

\author{
Ming Pei ${ }^{1,2 t}$, Junli Chen ${ }^{1,2 t}$, Shuo Dong ${ }^{3}$, Bo Yang ${ }^{1}$, Kang Yang ${ }^{1}$, Lijuan Wei ${ }^{1}$, \\ Jingbo Zhai $^{3 *}$ and Hongtao Yang ${ }^{1,2 *}$
}

${ }^{1}$ Division of Nephrology, First Teaching Hospital of Tianjin University of Traditional Chinese Medicine, Tianjin, China, ${ }^{2}$ National Clinical Research Center for Chinese Medicine Acupuncture and Moxibustion, Tianjin, China, ${ }^{3}$ Institute of Traditional Chinese Medicine, Tianjin University of Traditional Chinese Medicine, Tianjin, China

Background: Insomnia is one of the common problems in patients with maintenance hemodialysis (MHD). Previous studies have reported the beneficial effects of auricular acupressure (AA) for insomnia in patients with MHD. This study aimed to critically evaluate the efficacy and safety of AA for insomnia in patients with MHD.

Methods: Web of Science, Embase, PubMed, Cochrane Library, Chinese Biomedical Database, Wanfang Data, Chinese Science and Technology Periodicals database, and China National Knowledge Infrastructure were systematically searched from inception to April 30, 2020, to identify any eligible randomized controlled trials. MHD patients with insomnia were included regardless of age, gender, nationality, or race. The experimental interventions included $A A$ alone or $A A$ combined with other therapies. The control interventions included placebo, no treatment, or other therapies. The primary outcome was sleep quality measured by the Pittsburgh Sleep Quality Index (PSQI). RevMan 5.3 software was used for statistical analysis.

Results: Eight studies involving 618 participants were included for statistical analysis. A meta-analysis showed no significant difference of PSQI global score after 8 weeks of AA treatment compared with estazolam $(\rho=0.70)$. Other narrative analyses revealed that PSQI global score was significantly attenuated after AA treatment in comparison with mental health education $(p=0.03$, duration of 4 weeks; $p=0.02$, duration of 8 weeks), AA plus routine nursing care compared with routine nursing care alone $(p<0.0001)$, and AA plus footbath compared with footbath alone $(p=$ $0.01)$, respectively. A meta-analysis showed that $A A$ could significantly increase the response rate (reduction of PSQI global score by $25 \%$ and more) in comparison with estazolam $(p=0.01)$. Other narrative analyses reported that the response rate was significantly increased after AA treatment compared with sham AA $(p=$ 0.02), AA compared with mental health education $(p=0.04)$, and AA plus routine nursing care compared with routine nursing care alone $(p=0.0003)$, respectively. 
Conclusion: The present findings suggest that AA may be an alternative treatment for insomnia in patients with MHD. However, more large-scale, high-quality trials are still warranted to confirm these outcomes.

Keywords: auricular acupressure, insomnia, maintenance hemodialysis, systematic review, meta-analysis

\section{INTRODUCTION}

Insomnia is one of the common problems in patients with maintenance hemodialysis (MHD) (1-3). Insomnia may be caused by a variety of factors, including restlessness, pain, long duration on dialysis, and unhealthy lifestyle behavior $(2,4,5)$, which might potentially exert a negative impact on quality of life in dialysis patients (6).

Therapies for insomnia include both pharmacologic (e.g., benzodiazepines, non-benzodiazepine hypnotics) and nonpharmacologic treatments (e.g., cognitive behavioral therapies) (7). However, there is no specific recommendation for insomnia management in MHD patients according to the present clinical practice guidelines (7-10). Moreover, attention should also be paid to the barriers and risks of above interventions. For instance, benzodiazepine treatment may lead to Alzheimer's disease or stroke $(11,12)$. The effective administration of cognitive behavioral therapy might be limited in dialysis patients due to low patient adherence, lack of trained therapists, etc. (13). Therefore, it is urgently needed to seek alternative treatments.

Complementary and alternative medicine (CAM) has been applied for insomnia worldwide (e.g., in the United States) (14). Auricular acupressure (AA) is defined as a non-invasive technique in CAM to treat certain diseases or to relieve certain symptoms by taping drugs (e.g., semen vaccariae) or magnetic pellets, followed by application of physical pressure at specific acupoints on the ears $(15,16)$.

Several previous reviews have summarized the efficacy of AA for insomina. For instance, a systematic review indicated that AA might be effective for comorbid insomnia (17). Another meta-analysis showed that AA exerted a beneficial effect on the management of primary insomnia (18). Moreover, a few clinical studies have investigated the efficacy of AA for insomnia in MHD patients. For example, a pilot study revealed that AA could improve sleep quality in MHD patients with severe insomnia (16). Another trial indicated that AA was more effective than sham AA for treating insomnia in MHD patients (19). However, the evidence on AA for insomnia in MHD patients is still inconclusive due to the lack of high-quality systematic reviews.

Therefore, this study aimed to critically evaluate the efficacy and safety of AA for insomnia in patients with MHD.

\section{METHODS}

This study was performed according to the Preferred Reporting Items for Systematic Reviews and Meta-Analyses (PRISMA) (20). This study was registered on PROSPERO (No. CRD42020166054).

\section{Search Strategy}

We systematically searched Web of Science, Embase, PubMed, Cochrane Library, Chinese Biomedical Database (CBM), Wanfang Data, Chinese Science and Technology Periodicals database (VIP), and China National Knowledge Infrastructure (CNKI) from inception to April 30, 2020, without restriction on language or publication period. The search terms included (hemodialysis OR haemodialysis OR hemofiltration OR haemofiltration OR hemodiafiltration OR haemodiafiltration OR dialysis) AND (sleep OR insomnia OR wakeful OR sleepless OR "Early Awakening") AND (auricular OR acupressure OR acupoint OR auriculotherapy) AND (randomized OR randomly OR controlled OR placebo OR trial). Moreover, references of eligible studies were also manually searched to identify additional eligible studies.

\section{Inclusion and Exclusion Criteria Types of Studies}

Randomized controlled trials (RCTs) were included. Quasirandomized RCTs were excluded.

\section{Types of Patients}

MHD patients with insomnia were included, without restriction on age, gender, nationality, or race. The diagnosis of insomnia was in line with the internationally accepted diagnostic criteria, such as the 10th edition of International Classification of Diseases (ICD-10) and the Diagnostic and Statistical Manual of Mental Disorders fourth edition-Text Revision (DSM-IV-TR).

\section{Types of Interventions}

The experimental interventions included AA alone or AA combined with other therapies. The control interventions included placebo, no treatment, or other therapies. The duration and frequency of AA were unrestricted.

\section{Types of Outcomes}

The primary outcome was sleep quality measured by the Pittsburgh Sleep Quality Index (PSQI). To be specific, PSQI is a self-assessment questionnaire with a global score of 0 to 21 (21), where higher PSQI scores indicate worse sleep quality.

The secondary outcomes included response rate, use of sleep medications, and adverse events. Response was defined based on the reduction of PSQI global score, such as reduction of PSQI global score by $25 \%$ or more. Response rate refers to a ratio of the number of patients labeled as "response" divided by the number of all patients in a certain group.

\section{Selection of Studies}

First, titles and abstracts of potentially eligible studies were independently reviewed by two investigators (JC and MP) 
to delete duplicates and obviously irrelevant publications. Afterwards, full texts of the remaining studies were checked for final inclusion. Discrepancies were resolved through discussion or consultation with another investigator (JZ).

\section{Data Extraction}

The following information was extracted by two investigators (BY and KY): (1) study features (e.g., authors, year of publication, and sample size); (2) patient characteristics (e.g., diagnostic criteria for insomnia); (3) interventions (e.g., frequency, duration of treatment); and (4) outcome measurements (e.g., PSQI, response rate, use of sleep medications, and adverse events). If necessary, we attempted to contact authors by e-mail to obtain missing information.

\section{Quality Assessment}

Cochrane's risk of bias assessment tool was used to evaluate the methodological quality by two independent investigators (SD and MP). It includes seven items: random sequence generation, allocation concealment, blinding of personnel and participants, blinding of outcome measurement, incomplete outcome data, selective reporting, and other sources of bias (22). Risk of bias in each item is categorized into three levels, namely, low, high, or unclear.

\section{Statistical Analysis}

RevMan 5.3 software was utilized for statistical analysis. Risk ratio (RR) with 95\% confidence intervals (CIs) was used for the dichotomous variables. Mean difference (MD) with $95 \%$ CIs was calculated for the continuous variables. Chi-square test and $I^{2}$ statistics were employed to investigate the heterogeneity across studies. In the case of $p>0.10$ or $I^{2}<50 \%$, a fixedeffect model was used to estimate pooled effect; otherwise, a random-effect model was used. Moreover, funnel plot was used to examine publication bias of the meta-analysis including 10 or more studies. If necessary, subgroup or narrative analysis was conducted based on the therapeutic duration, comparisons, etc.

\section{RESULTS}

\section{Literature Search}

A total of 187 articles were identified in the initial search. After excluding duplicates, 92 articles remained. Thirty-six irrelevant articles were subsequently eliminated after screening titles and abstracts. Full texts of the remaining articles were assessed for eligibility. Finally, eight studies were included for statistical analysis (19, 23-29). The screening process is shown in Figure 1.

\section{Study Characteristics}

The characteristics of included studies are presented in Table $\mathbf{1}$. To be specific, the eight studies were published from 2008 to 2019, involving 618 participants (322 in the experimental group and 296 in the control group). The sample size ranged from 20 to 45 in each group. Six studies used Chinese Classification of Mental Disorders (CCMD) to diagnose insomnia. One study used ICD-10 and CCMD-3 to diagnose insomnia. Another one used DSM-IV-TR to diagnose insomnia. The interventions in

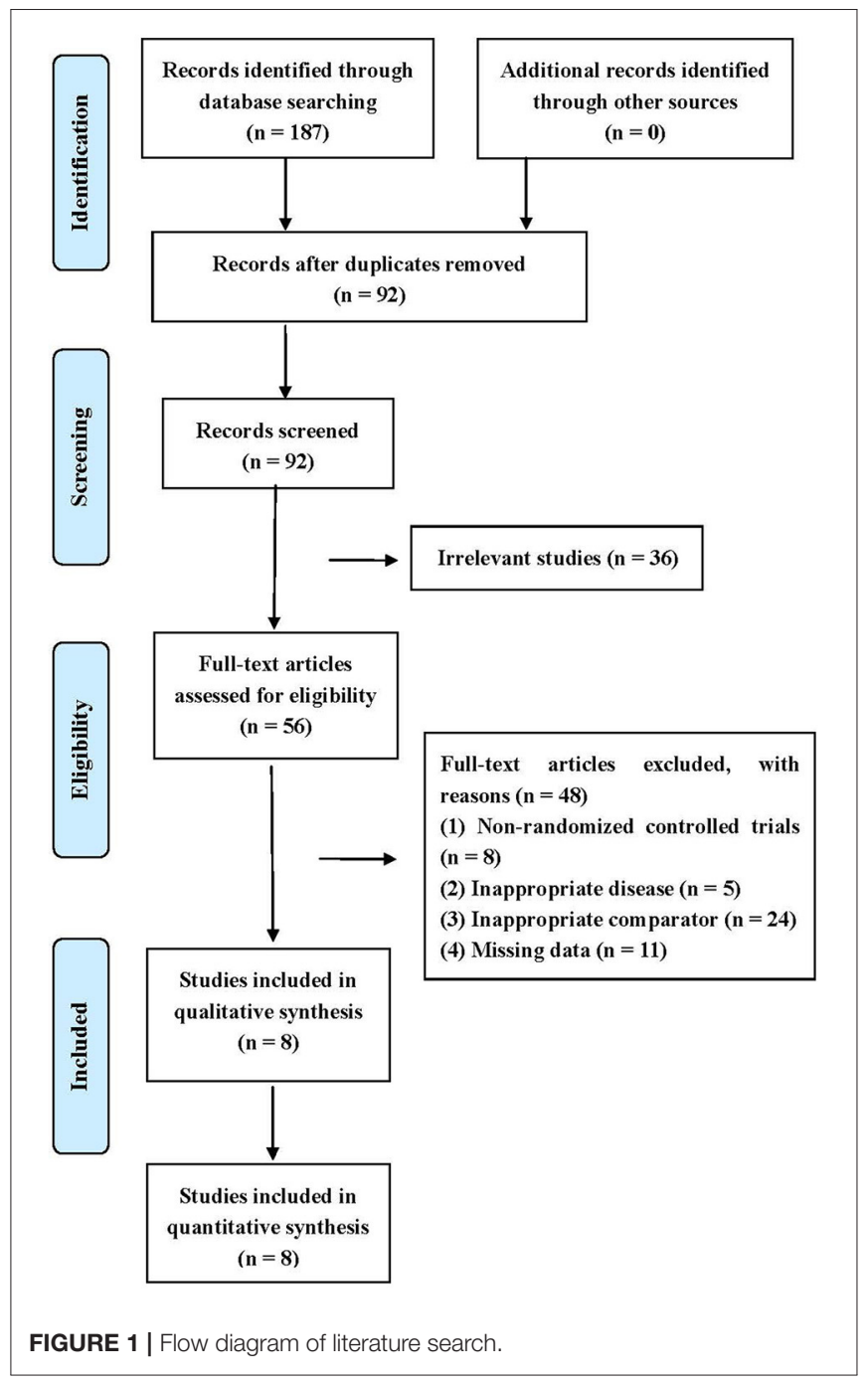

the experimental group included AA, AA plus routine nursing care, and AA plus footbath. The interventions in the control group included sham AA, estazolam, routine nursing care, and footbath. Duration of treatment ranged from 4 weeks to 2 months. The indicators of outcome included PSQI score, response rate, use of sleep medications, and adverse reactions.

\section{Quality Assessment}

The outcomes of risk of bias assessment are summarized in Figures 2, 3. A random number table was used for participant assignment in four of the included studies $(19,26,28,29)$, while the specific randomized allocation method was not described in other studies. Only one study reported allocation concealment and blinding (19). Complete outcome data were available in all studies. One study was graded low risk of bias in reporting bias (19). The risk of bias in other bias was graded unclear in all included studies.

\section{PSQI Score}

Six included studies reported PSQI global score (19, 23, 25$27,29)$. Due to the heterogeneity of therapeutic duration and 
TABLE 1 | Characteristics of included studies.

\begin{tabular}{|c|c|c|c|c|c|c|c|c|}
\hline Article & $\begin{array}{l}\text { Diagnostic } \\
\text { criteria for } \\
\text { insomnia }\end{array}$ & $\begin{array}{l}\text { Sample size in } \\
\text { the experimental } \\
\text { group }\end{array}$ & $\begin{array}{l}\text { Sample size in } \\
\text { the control } \\
\text { group }\end{array}$ & $\begin{array}{l}\text { Intervention(s) in } \\
\text { the experimental } \\
\text { group }\end{array}$ & $\begin{array}{l}\text { Intervention(s) in the } \\
\text { control group }\end{array}$ & Frequency of AA & $\begin{array}{l}\text { Duration of } \\
\text { treatment }\end{array}$ & Outcomes \\
\hline Zhang (23) & ICD-10,CCMD-3 & 20 & $\begin{array}{l}20 \text { in each control } \\
\text { group }\end{array}$ & AA & $\begin{array}{l}\text { Estazolam in control group } \\
\text { (1); mental health education } \\
\text { in control group (2) }\end{array}$ & Four to five times a day & 8 weeks & PSQI, Response rate \\
\hline Zhao (24) & CCMD-3 & 30 & 30 & AA & Estazolam & Four times a day & 8 weeks & Response rate \\
\hline Zhou et al. (25) & CCMD-3 & 30 & 30 & AA & Estazolam & Four to five times a day & 1 month & PSQI, Response rate \\
\hline Zheng and Liu (26) & CCMD-2 & 40 & 40 & AA & Estazolam & Not reported & 8 weeks & PSQI \\
\hline Zou et al. (19) & DSM-IV-TR & 32 & 31 & AA & Sham AA & $\begin{array}{l}\text { Three to five times a } \\
\text { day }\end{array}$ & 8 weeks & $\begin{array}{l}\text { PSQI, Response rate, } \\
\text { Use of sleep } \\
\text { medications, adverse } \\
\text { reactions }\end{array}$ \\
\hline Yin (27) & CCMD-2 & $\begin{array}{l}45 \text { in each } \\
\text { experimental } \\
\text { group }\end{array}$ & 45 & $\begin{array}{l}\text { AA in experimental } \\
\text { group (1); } A A+ \\
\text { foot bath in } \\
\text { experimental } \\
\text { group (2) }\end{array}$ & Foot bath & Not reported & 4 weeks & $\begin{array}{l}\text { PSQI, adverse } \\
\text { reactions }\end{array}$ \\
\hline Huang et al. (28) & CCMD & 40 & 40 & AA & Estazolam & Four to five times a day & 2 months & Response rate \\
\hline Li and Zhang (29) & CCMD & 40 & 40 & $\begin{array}{l}\mathrm{AA}+\text { routine } \\
\text { nursing care }\end{array}$ & $\begin{array}{l}\text { Routine nursing care (health } \\
\text { education, emotional } \\
\text { support, etc.) }\end{array}$ & $\begin{array}{l}\text { Three to five times a } \\
\text { day }\end{array}$ & 4 weeks & PSQI, Response rate \\
\hline
\end{tabular}

ICD-10, International Classification of Diseases (10th edition); CCMD-2, Chinese Classification of Mental Disorders (2nd edition); CCMD-3, Chinese Classification of Mental Disorders (3rd edition); DSM-IV-TR, Diagnostic and Statistical Manual of Mental Disorders fourth edition-Text Revision; AA, auricular acupressure; PSQI, Pittsburgh Sleep Quality Index. 


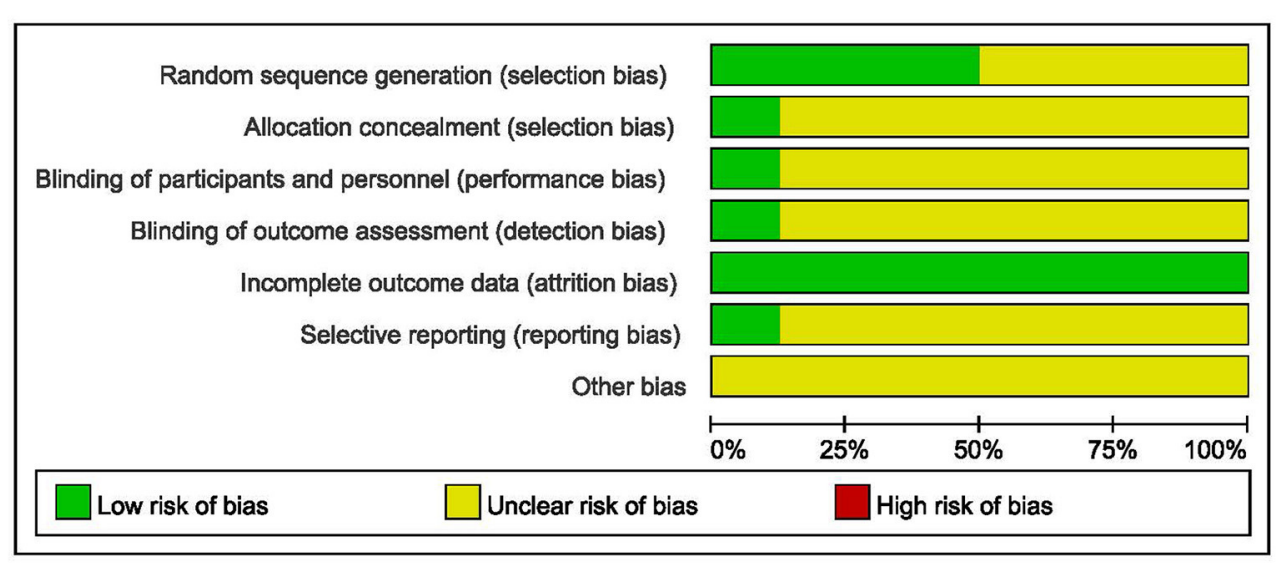

FIGURE 2 | Risk of bias graph.

comparisons, the subgroup or narrative analysis was conducted instead of the meta-analysis including all eligible studies.

As shown in Figure 4, there was no significant difference of PSQI global score after 4 weeks or 1 month of AA treatment compared with estazolam $(n=100, \mathrm{MD}=-1.15,95 \% \mathrm{CI}:-4.97$ to $2.68, p=0.56$ ), which was similar with the outcome of metaanalysis at the end of 8 weeks $(n=120, \mathrm{MD}=-0.64,95 \% \mathrm{CI}$ : -3.86 to $2.57, p=0.70$ ).

One study found that AA alone could significantly attenuate the PSQI global score compared with mental health education alone $(n=40, \mathrm{MD}=-1.80,95 \% \mathrm{CI}:-3.41$ to $-0.19, p=$ 0.03 , duration of 4 weeks; $n=40, \mathrm{MD}=-1.80,95 \% \mathrm{CI}:-3.26$ to $-0.34, p=0.02$, duration of 8 weeks) (23). Another study revealed that the PSQI global score was significantly decreased after 4-week administration of AA plus routine nursing care compared with routine nursing care alone $(n=80, \mathrm{MD}=-2.38$, 95\% CI: -3.46 to $-1.30, p<0.0001)$ (29). Moreover, a threearm clinical trial was conducted to assess the efficacy of AA, footbath, and a combination of both for insomnia in MHD patients (27). As a result, there was no significant difference between AA and footbath $(n=90, \mathrm{MD}=0.36,95 \% \mathrm{CI}:-0.92$ to $1.64, p=0.58$ ). However, AA combined with footbath could significantly attenuate the PSQI global score in comparison with footbath alone $(n=90, \mathrm{MD}=-1.36,95 \% \mathrm{CI}:-2.41$ to -0.31 , $p=0.01$ ). A pilot trial found that AA could decrease PSQI global score compared with sham AA, but without statistical significance $(n=63, \mathrm{MD}=-1.55,95 \% \mathrm{CI}:-3.56$ to $0.46, p=$ 0.13) (19).

\section{Response Rate}

Among the eight included studies, six of them reported the response rate $(19,23-25,28,29)$. The definition of response was various across these studies.

In three trials, response was defined as reduction of PSQI global score by $25 \%$ or more $(24,25,28)$. One study found no significant difference of response rate after 1-month treatment between AA and estazolam ( $n=60, \mathrm{RR}=1.23,95 \% \mathrm{CI}: 0.96$ to $1.57, p=0.10$, Figure 5) (25). The meta-analysis including two studies showed that AA alone could significantly increase response rate compared with estazolam alone at the end of 8 weeks or 2 months ( $n=140, \mathrm{RR}=1.21,95 \% \mathrm{CI}$ : 1.04 to 1.40 , $p=0.01$, Figure 5).

In another study, response was defined as reduction of PSQI global score by $20 \%$ or more (29). As a result, AA plus routine nursing care could significantly improve response rate in MHD patients compared with routine nursing care alone $(n=80, \mathrm{RR}$ $=2.20,95 \%$ CI: 1.44 to $3.36, p=0.0003)$. Another study defined response as reduction of PSQI global score by five points and more (23), which showed that AA could significantly increase response rate compared with mental health education $(n=$ $40, \mathrm{RR}=1.50,95 \% \mathrm{CI}: 1.02$ to $2.21, p=0.04$ ), However, no statistical difference of response rate was identified between AA and estazolam $(n=40, \mathrm{RR}=1.00,95 \% \mathrm{CI}: 0.81$ to $1.23, p$ $=1.00)$. Another trial defined response as reduction of PSQI global score by three points and more (19), which revealed that the response rate was significantly higher in the AA group than the sham AA group $(n=63, \mathrm{RR}=1.94,95 \% \mathrm{CI}: 1.09$ to 3.45 , $p=0.02)$.

\section{Use of Sleep Medications}

One study reported that the proportion of patients taking sleep medications was significantly reduced $(p<0.01)$ in the AA group, compared with the sham AA group after 8 weeks of treatment (19).

\section{Adverse Reactions}

Two studies described the adverse reaction. One study showed no adverse reactions in the AA or sham AA group (19), while another study revealed no adverse reactions in the AA plus footbath or footbath alone group (27).

\section{Publication Bias}

Publication bias was not assessed because no meta-analyses included over 10 studies. 


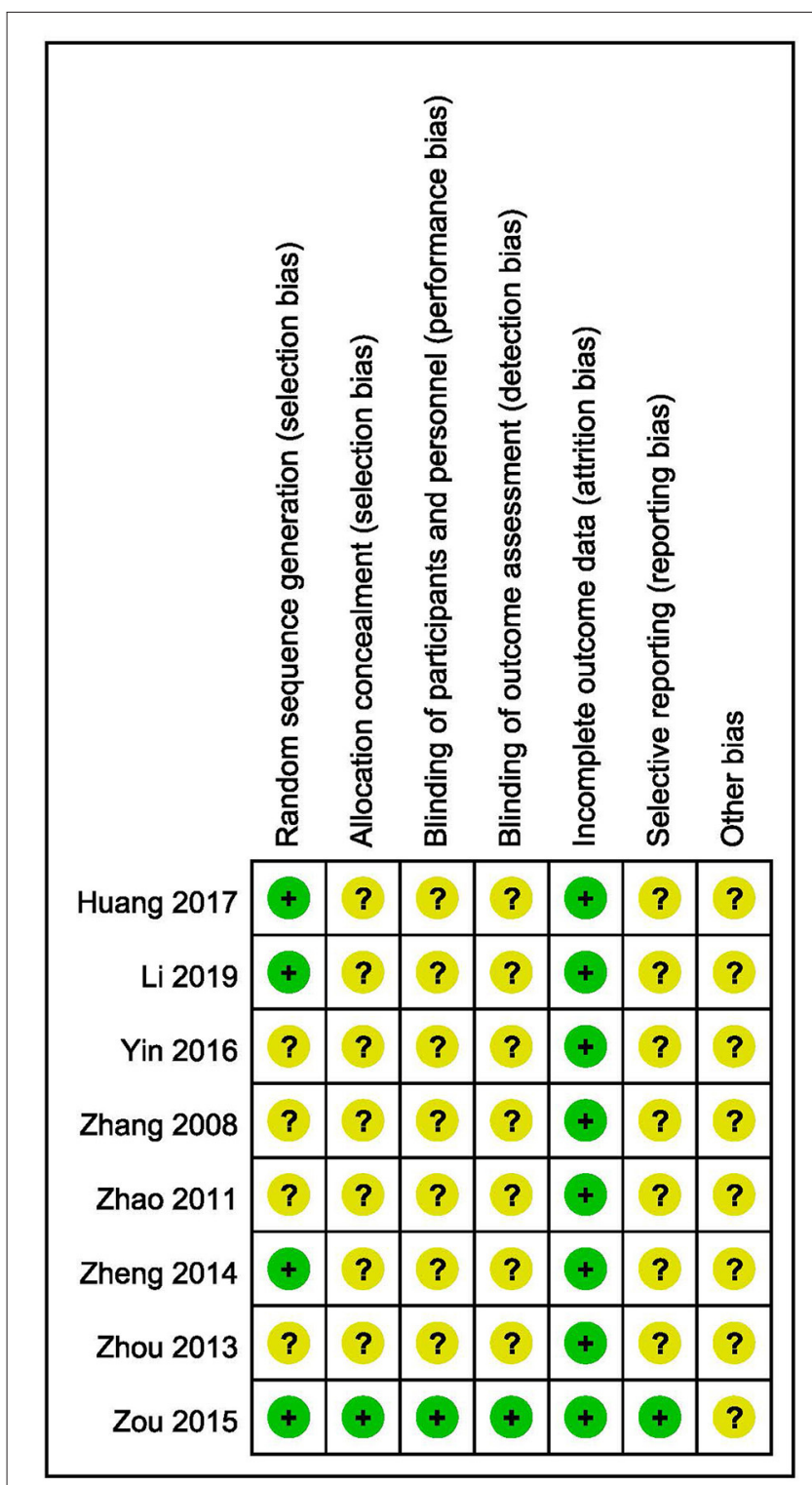

FIGURE 3 | Risk of bias summary.

\section{DISCUSSION}

\section{Main Findings and Interpretation}

In this study, we critically evaluated the efficacy and safety of AA for insomnia in MHD patients.

A pilot study showed no statistical difference in PSQI global score, but significant difference in response rate between AA and sham AA (19). Power is defined as the probability of rejecting a false null hypothesis (30). It is generally assumed to be $80 \%$ to $90 \%$ when calculating sample size (30). In this pilot study, however, the sample size was not calculated. Herein, we calculated the power of these two indicators using the results by PASS software, which showed a power of 32 and $67 \%$, respectively, to detect a superiority margin difference between
AA and sham AA. They might be insufficient to reject a false null hypothesis. Therefore, high-quality trials with a larger sample size are further warranted.

A clinical practice guideline in 2017 reported that estazolam (a benzodiazepine derivative) could significantly improve sleep duration compared with placebo (9). Estazolam has also been approved to treat insomnia in the United States by the Food and Drug Administration (7). However, a study found that benzodiazepines were associated with risk of any fracture (RR $=1.31, p=0.03$ ) in hemodialysis patients (31). Another study demonstrated that benzodiazepines may be associated with greater mortality in incident dialysis patients (32). Our meta-analyses showed no significant difference of PSQI global score between AA and estazolam. However, these meta-analyses had some defects, such as high statistical heterogeneity $\left(I^{2}\right.$ $>90 \%$ ), small sample size, and a small number of included studies. We found that one study with a small sample size reported no significant difference of response rate after 1-month treatment between AA and estazolam. However, a meta-analysis involving another two studies showed that response rate was significantly higher after 8 weeks or 2 months of AA treatment compared with estazolam. One study reported that AA of 8 weeks' duration could significantly improve patient comfort level measured by Maintenance Hemodialysis Patients Comfort Scale compared with estazolam (24). No significant difference or inconsistent results may be associated with multiple factors, such as severity of insomnia, small sample size, and cumulative effect. It is important to use double-blind and double-dummy technology in trials comparing two interventions, which can reduce performance bias, detection bias, etc. However, only one study reported blinding (19). Double-dummy technology was not reported in included studies. AA includes multiple steps, such as selecting acupoints, taping drugs or magnetic pellets, and physical pressure. These steps may be various across included studies. For example, Huang et al. taped semen vaccariae on patients' ears (28). Magnetic pellet was used in another study (24). They may partly explain the above results. The safety of AA vs. estazolam for insomnia in MHD patients was not assessed due to a lack of information. In addition, the efficacy and safety of AA vs. other drugs for insomnia in MHD patients were also not investigated due to the absent trials on this topic.

Some clinical guidelines reported that psychological and behavioral therapies were used for the management of insomnia $(7,33)$. In this review, only one enrolled study found that AA combined with routine nursing care (a combination of health education, emotional support, etc.) could significantly relieve insomnia symptoms in MHD patients in comparison with routine nursing care alone (29). Several previous studies reported that footbath could improve sleep quality $(34,35)$. One included study showed that AA combined with footbath could significantly attenuate the PSQI global score compared with footbath alone (27), indicating that a combination of AA and routine nursing care/footbath may be a beneficial therapeutic strategy for insomnia management in MHD patients. However, the efficacy of AA combined with drugs such as estazolam for insomnia in MHD patients was not assessed due to a lack of relevant trials in this study. 


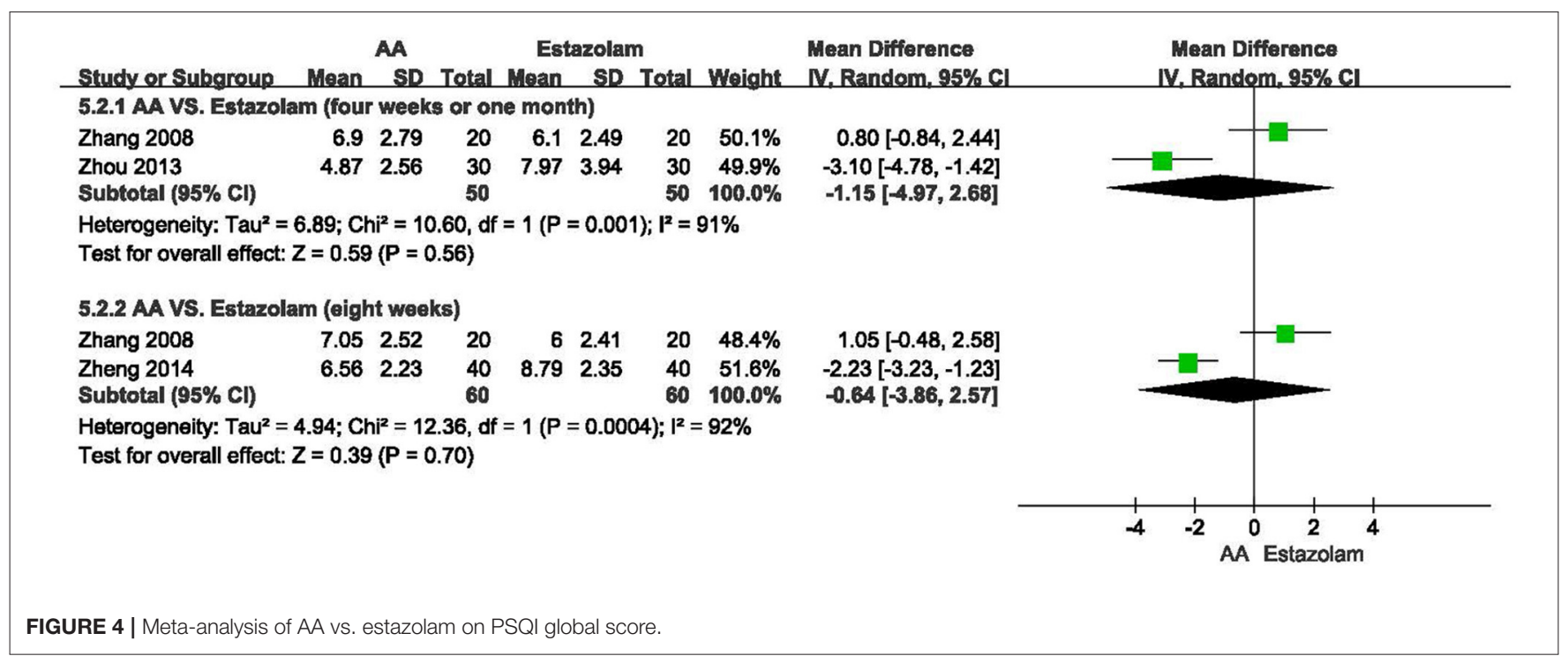

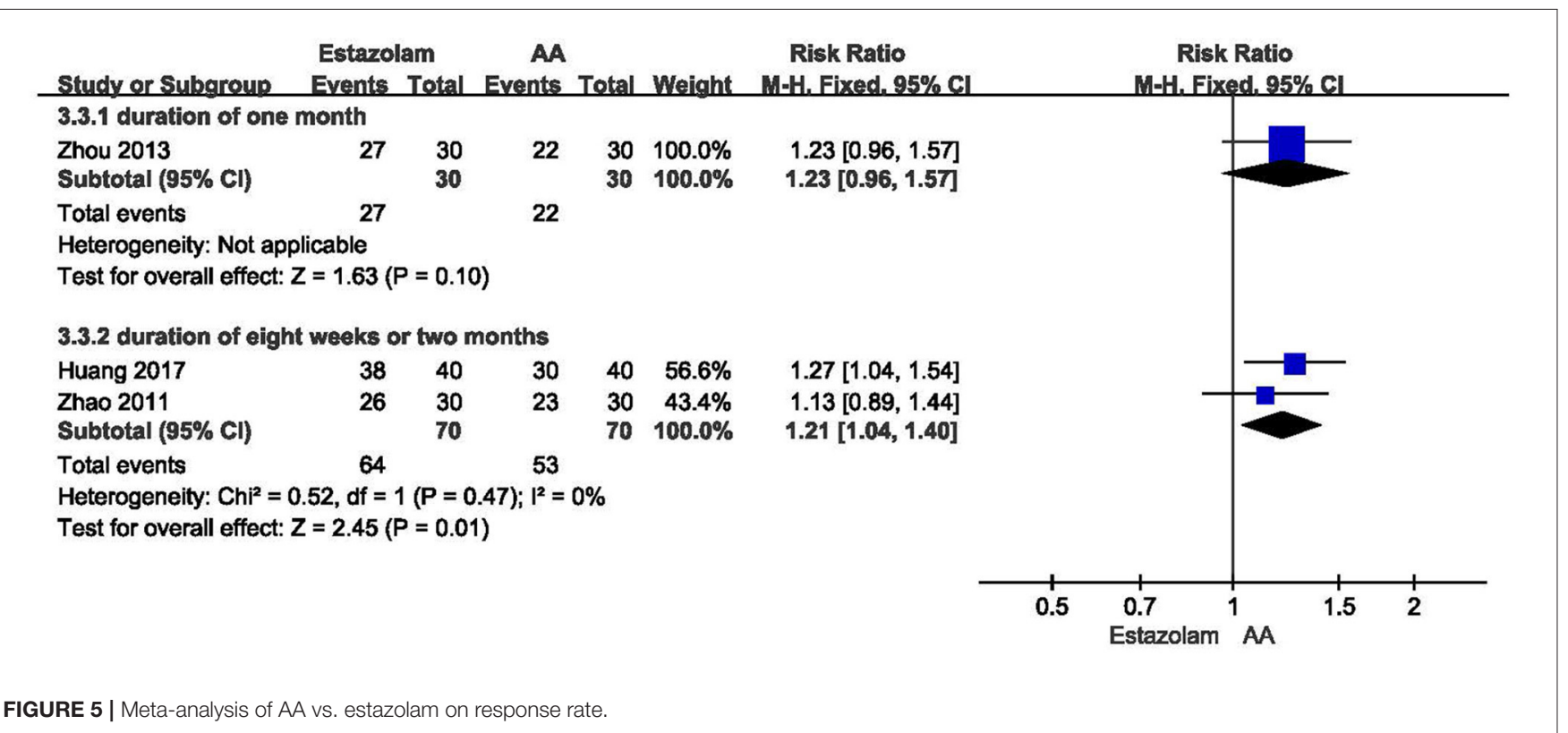

\section{Limitations}

Several potential limitations should be taken into consideration. To begin with, a small sample size may decrease the probability of rejecting a false null hypothesis. Secondly, performing the meta-analysis was limited due to the heterogeneity of duration, definition of response rate, etc. Thirdly, the reported outcome measurement varied across studies. No core outcome set on this topic was published in Core Outcome Measures in Effectiveness Trials (COMET). Therefore, it is necessary to establish a core outcome set on this topic. Fourthly, the methodological quality of included studies may be reduced due to unclear risk of bias of allocation concealment and blinding item in most of the included studies. Fifthly, only two studies reported adverse reactions. Therefore, the evidence is insufficient to evaluate the safety of AA for insomnia in MHD patients. Finally, the causes of insomnia were unrestricted in this study. Only one study reported the causes of insomnia, but did not provide the efficacy of AA according to different causes of insomnia (23). Therefore, no subgroup analysis based on causes of insomnia was conducted because of insufficient information.

\section{CONCLUSION}

The present findings in our study suggest that AA may be an alternative treatment for insomnia in patients with MHD. However, more large-scale, high-quality trials are still warranted to confirm these outcomes. 


\section{DATA AVAILABILITY STATEMENT}

The original contributions generated for this study are included in the article/supplementary material, further inquiries can be directed to the corresponding author/s.

\section{AUTHOR CONTRIBUTIONS}

JZ, MP, and HY conceived the study. JC designed the search strategy. JC and MP screened the studies. BY and KY extracted the data. SD and MP assessed the methodological quality. LW

\section{REFERENCES}

1. Sabbatini M, Minale B, Crispo A, Pisani A, Ragosta A, Esposito R, et al. Insomnia in maintenance haemodialysis patients. Nephrol Dial Transplant. (2002) 17:852-6. doi: 10.1093/ndt/17.5.852

2. Merlino G, Piani A, Dolso P, Adorati M, Cancelli I, Valente M, et al. Sleep disorders in patients with end-stage renal disease undergoing dialysis therapy. Nephrol Dial Transplant. (2006) 21:184-90. doi: 10.1093/ndt/ gfil44

3. Rai M, Rustagi T, Rustagi S, Kohli R. Depression, insomnia and sleep apnea in patients on maintenance hemodialysis. Indian J Nephrol. (2011) 21:2239. doi: 10.4103/0971-4065.83028

4. Hamzi MA, Hassani K, Asseraji M, El Kabbaj D. Insomnia in hemodialysis patients: a multicenter study from morocco. Saudi J Kidney Dis Transpl. (2017) 28:1112-8. doi: 10.4103/1319-2442.215152

5. Scherer JS, Combs SA, Brennan F. Sleep disorders, restless legs syndrome, and uremic pruritus: diagnosis and treatment of common symptoms in dialysis patients. Am J Kidney Dis. (2017) 69:117-28. doi: 10.1053/j.ajkd.2016. 07.031

6. Theofilou P. Association of insomnia symptoms with kidney disease quality of life reported by patients on maintenance dialysis. Psychol Health Med. (2013) 18:70-8. doi: 10.1080/13548506.2012.674144

7. Qaseem A, Kansagara D, Forciea MA, Cooke M, Denberg TD, and Clinical Guidelines Committee of the American College of Physicians. Management of chronic insomnia disorder in adults: a clinical practice guideline from the American College of Physicians. Ann Intern Med. (2016) 165:12533. doi: 10.7326/M15-2175

8. Riemann D, Baglioni C, Spiegelhalder K, Bassetti C, Bjorvatn B, Dolenc Groselj L, et al. European guideline for the diagnosis and treatment of insomnia. J Sleep Res. (2017) 26:675-700. doi: 10.1111/jsr.12594

9. Sateia MJ, Buysse DJ, Krystal AD, Neubauer DN, Heald JL. Clinical practice guideline for the pharmacologic treatment of chronic insomnia in adults: an American academy of sleep medicine clinical practice guideline. J Clin Sleep Med. (2017) 13:307-49. doi: 10.5664/jcsm.6470

10. Wilson S, Anderson K, Baldwin D, Dijk DJ, Espie A, Espie C, et al. British Association for Psychopharmacology consensus statement on evidence-based treatment of insomnia, parasomnias and circadian rhythm disorders: an update. J Psychopharmacol. (2019) 33:923-47. doi: 10.1177/0269881119855343

11. Billioti de Gage S, Moride Y, Ducruet T, Kurth T, Verdoux H, Tournier M, et al. Benzodiazepine use and risk of Alzheimer's disease: case-control study. BMJ. (2014) 349:g5205. doi: 10.1136/bmj.g5205

12. Huang WS, Muo $\mathrm{CH}$, Chang $\mathrm{SN}$, Chang $\mathrm{YJ}$, Tsai $\mathrm{CH}$, Kao $\mathrm{CH}$. Benzodiazepine use and risk of stroke: a retrospective population-based cohort study. Psychiatry Clin Neurosci. (2014) 68:255-62. doi: 10.1111/pc n. 12117

13. Flythe JE, Hilliard T, Lumby E, Castillo G, Orazi J, Abdel-Rahman EM, et al. Fostering innovation in symptom management among hemodialysis patients: paths forward for insomnia, muscle cramps, and fatigue. Clin J Am Soc Nephrol. (2019) 14:150-60. doi: 10.2215/CJN.07670618

14. Pearson NJ, Johnson LL, Nahin RL. Insomnia, trouble sleeping, and complementary and alternative medicine: analysis of the 2002 performed the statistical analysis. MP, JC, SD, BY, KY, LW, JZ, and HY drafted the manuscript and reviewed and revised the manuscript. All authors have read and approved the final version of the manuscript.

\section{FUNDING}

This study was supported by the National Natural Science Foundation of China (Nos. 81703860 and 81673909) and the National Key Research and Development Program of China (No. 2019YFC1709401). national health interview survey data. Arch Intern Med. (2006) 166:1775-82. doi: 10.1001/archinte.166.16.1775

15. Gao H, Zhang L, Liu J. Auricular acupressure for myopia in children and adolescents: a systematic review. Complement Ther Clin Pract. (2020) 38:101067. doi: 10.1016/j.ctcp.2019.101067

16. Wu Y, Zou C, Liu X, Wu X, Lin Q. Auricular acupressure helps improve sleep quality for severe insomnia in maintenance hemodialysis patients: a pilot study. J Altern Complement Med. (2014) 20:35663. doi: $10.1089 / \mathrm{acm} .2013 .0319$

17. Zhao H, Li D, Yang Y, Liu Y, Li J, Mao J. Auricular plaster therapy for comorbid insomnia: a systematic review and meta-analysis of randomized controlled trials. Evid Based Complement Alternat Med. (2019) 2019:7120169. doi: 10.1155/2019/7120169

18. Lan $\mathrm{Y}, \mathrm{Wu} \mathrm{X}, \mathrm{Tan} \mathrm{HJ}$, Wu N, Xing JJ, Wu FS, et al. Auricular acupuncture with seed or pellet attachments for primary insomnia: a systematic review and meta-analysis. BMC Complement Altern Med. (2015) 15:103. doi: 10.1186/s12906-015-0606-7

19. Zou C, Wu Y, Su G, Wu X, Liu X, Lin Q, et al. Auricular acupressure on specific points for hemodialysis patients with insomnia: a pilot randomized controlled trial. PLoS ONE. (2015) 10:e0122724. doi: 10.1371/journal.pone. 0122724

20. Moher D, Liberati A, Tetzlaff J, Altman DG, PRISMA Group. Preferred reporting items for systematic reviews and meta-analyses: the PRISMA statement. PLoS Med. (2009) 6:e1000097. doi: 10.1371/journal.pmed. 1000097

21. Wei CY, Chung TC, Wu SC, Chung CF, Wu WP. The subjective sleep quality and heart rate variability in hemodialysis patients. Ren Fail. (2011) 33:109-17. doi: 10.3109/0886022X.2010.541578

22. Higgins JPT, Thomas J, Chandler J, Cumpston M, Li T, Page MJ, et al. Cochrane Handbook for Systematic Reviews of Interventions version 6.2 (updated February 2021). Cochrane. (2021). Available online at: www.training.cochrane.org/handbook

23. Zhang L. A Clinical Study on Auricular Acupressure for Insomnia in Patients With Maintenance Hemodialysis. Dissertation/Master's Thesis. Wuhan (Hubei): Hubei College of Traditional Chinese Medicine (2008).

24. Zhao TL. The Effect Study of Auricular Point Magnetic Bead Plaster Therapy on Sleep Disorders and Comfort Status in Maintenance Hemodialysis Patients. Dissertation/Master's Thesis. Fuzhou (Fujian): Fujian University of Traditional Chinese Medicine (2011).

25. Zhou M, Lin QZ, Wu XQ. Intervention affects of auricular plaster therapy on sleep disturbance in maintenance hemodialysis patients. CJTCMP. (2013) 28:855-7. Available online at: http://en.cnki.com.cn/Article_en/ CJFDTOTAL-BXYY201303080.htm

26. Zheng QH, Liu CF. Influence of auricular acupoint bean pressing on insomnia and life quality in hemodialysis patients. Chin Med Modern Dist Educ China. (2014) 12:16-8. doi: 10.3969/j.issn.1672-2779.2014.20.007

27. Yin CL. Auricular acupressure combined with footbath for insomnia in forty-five patients with maintenance hemodialysis. Chin J Trad Med Sci Technol. (2016) 23:486-7. Available online at: http://www.cnki.com.cn/ Article/CJFDTotal-TJYY201604053.htm

28. Huang $\mathrm{YX}, \mathrm{Wu} \mathrm{AQ}$, Mo HM. A clinical observation of Auricular acupressure for insomnia in patients with maintenance hemodialysis. $J$ 
Pract Trad Chin Med. (2017) 33:165-6. doi: 10.3969/j.issn.1004-2814.2017. 02.041

29. Li AY, Zhang JY. A clinical observation of auricular acupressure for relieving insomnia in patients with maintenance hemodialysis. World Latest Med Inform. (2019) 19:258-60.

30. In J. Considerations when calculating the sample size for an inequality test. Korean J Anesthesiol. (2016) 69:327-31. doi: 10.4097/kjae.2016.69. 4.327

31. Jadoul M, Albert JM, Akiba T, Akizawa T, Arab L, Bragg-Gresham JL, et al. Incidence and risk factors for hip or other bone fractures among hemodialysis patients in the Dialysis Outcomes and Practice Patterns Study. Kidney Int. (2006) 70:1358-66. doi: 10.1038/sj.ki.5001754

32. Winkelmayer WC, Mehta J, Wang PS. Benzodiazepine use and mortality of incident dialysis patients in the United States. Kidney Int. (2007) 72:138893. doi: 10.1038/sj.ki.5002548

33. Schutte-Rodin S, Broch L, Buysse D, Dorsey C, Sateia M. Clinical guideline for the evaluation and management of chronic insomnia in adults. J Clin Sleep Med. (2008) 4:487-504. doi: 10.5664/jcsm. 27286
34. Kim HJ, Lee Y, Sohng KY. The effects of footbath on sleep among the older adults in nursing home: a quasi-experimental study. Complement Ther Med. (2016) 26:40-6. doi: 10.1016/j.ctim.2016.02.005

35. Chiu HY, Lin EY, Chiu HT, Chen PY. A feasibility randomized controlled crossover trial of home-based warm footbath to improve sleep in the chronic phase of traumatic brain injury. J Neurosci Nurs. (2017) 49:3805. doi: 10.1097/JNN.0000000000000325

Conflict of Interest: The authors declare that the research was conducted in the absence of any commercial or financial relationships that could be construed as a potential conflict of interest.

Copyright (C) 2021 Pei, Chen, Dong, Yang, Yang, Wei, Zhai and Yang. This is an open-access article distributed under the terms of the Creative Commons Attribution License (CC BY). The use, distribution or reproduction in other forums is permitted, provided the original author(s) and the copyright owner(s) are credited and that the original publication in this journal is cited, in accordance with accepted academic practice. No use, distribution or reproduction is permitted which does not comply with these terms. 